\title{
PachyonychiaCongenita: A Case Report
}

\author{
Marwa A. Abo Elmagd, and Rasha I. Mohamed \\ Department of Dermatology, Venereology and Andrology,Faculty of Medicine, \\ Sohag University
}

\section{Introduction}

Pachyonychiacongenita $(\mathrm{PC})$ is a rare autosomal dominant disorder of keratinization. ${ }^{[1]}$ It was first documented by Muller in $1904^{[2]}$ followed by Jadassohn and Lewandowsky in $1906 .{ }^{[3]}$ It is classified into four types, of which the two important ones include type-1 (Jadassohn-Lewandowsky type) and type-2 (Jackson-Lawler type). These are characterized by subungual hyperkeratosis, focal palmoplantarkeratoderma, oral leukokeratosis, which are usually

\section{Case Report}

An 7-year-old boy born of consanguineous parentage, with normal developmental milestones for his age exceptfor natal teeth ; also presented with nail defects since birth along with numerous skin lesions. Family history was unremarkable. Cutaneous examination revealed dystrophic, discolored, and thickened toenails and one fingernail along with massive subungual hyperkeratosis producing a distal elevation of nail

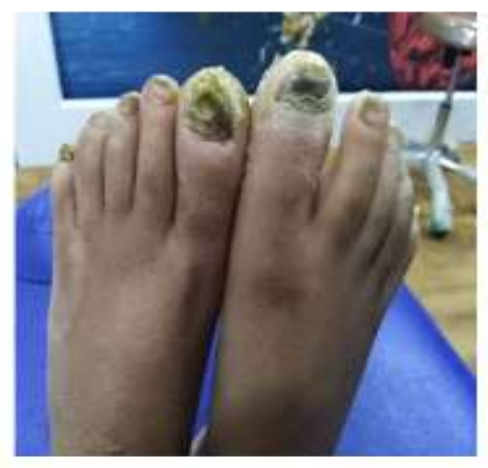

Figure 1

Dystrophic toe nails with subungual hyperkeratosis plates and wedge-shaped deformity of the nails. This resulted in the upward growth of the distal edge of the nail plates[Figure 1],[Figure 2], [Figure 3]. Besides, there were numerous, hyperkeratotic lesions over the entire body, concentrated over bothknees,legs and flanks [Figure 4],[Figure 5],. Marked hyperhidrosis of the palms and soles was observed. Palmoplantarkeratoderma was present, along with painful ulcerated plaques[Figure 6]. Routine laboratory investigations including complete hemogram, hepatic profile, and renal profile were within normal limits. $\mathrm{KOH}$ microscopy and culture of nail clippings was negative. Skin biopsy from a hyperkeratotic lesion from the leg, showed orthokeratosis and acanthosis[Figure 71. No evidence of any malignancy was found during the thorough work up. Genetic and molecular biological studies could not be carried out due to lack of infrastructure facilities. Based on the above findings, he was diagnosed as pachonychiacongenita type II. 
SOHAG MEDICAL JOURNAL

Vol. 23 No.2 Apr 2019

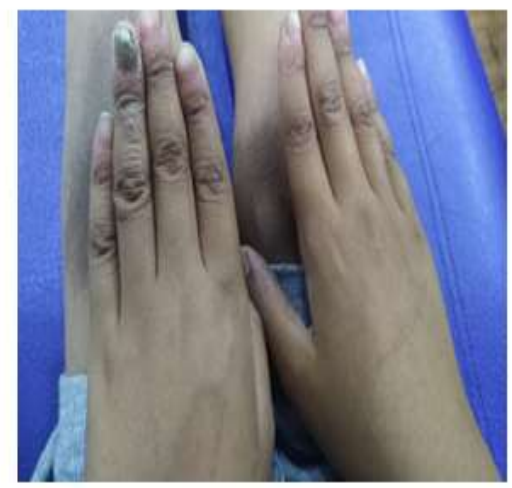

Figure 2 Dystrophic finger nail with wedge-shaped deformity

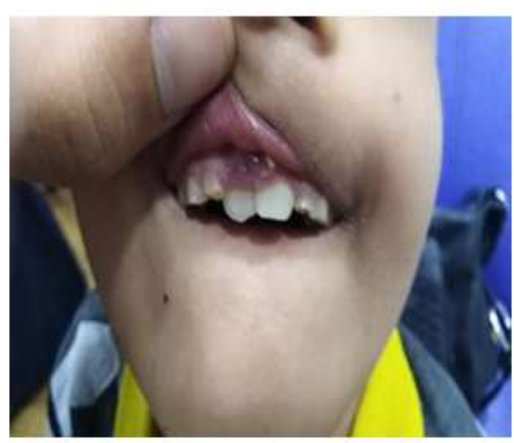

Figure 3 Natal teeth

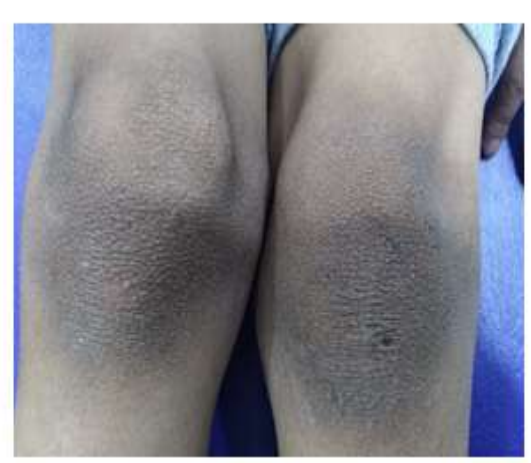

Figure 4

Follicular hyperkeratosis of knees
PachyonychiaCongenita: A Case Report Marwa A. Abo Elmagd,and Rasha I. Mohamed

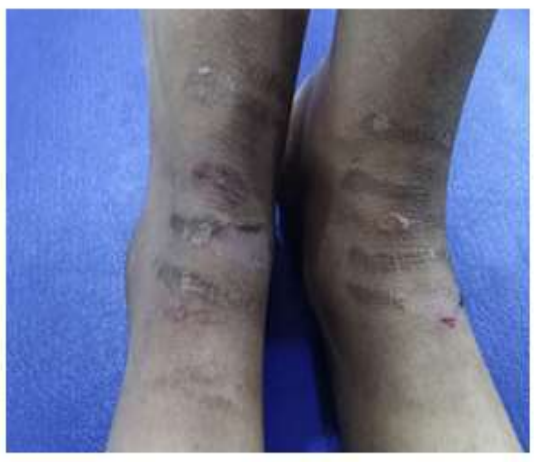

Figure 5

hyperkeratotic plaque

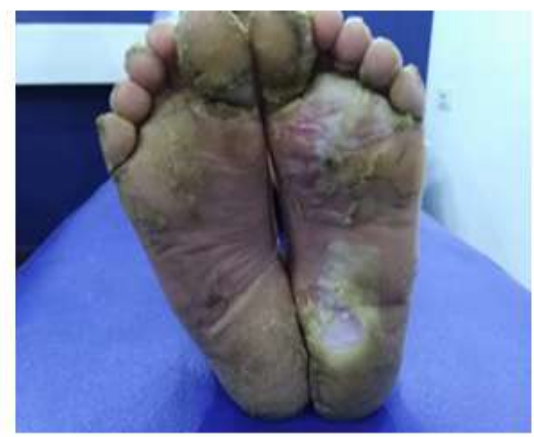

Figure 6

plantar keratoderma

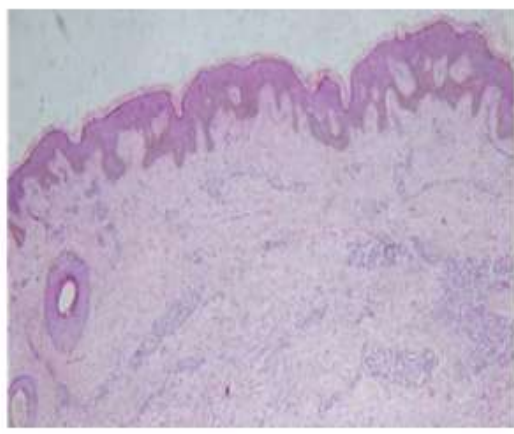

Figure 7

showing orthokeratosis and acanthosis ( $\mathrm{H}$ and $\mathrm{E}, \times 10$ ) 


\section{Discussion}

Pachyonychiacongenita (PC) is a rare, but wellcharacterized autosomal dominant disorder ofkeratinization characterized by a triad of sub-ungual hyperkeratosis with accumulation ofhard keratinous material beneath the distalportion of the nails, lifting the nails from thenail bed, keratosis palmaris et plantaris withthick callosities, especially on the soles andthick white areas on the oral mucosa[5]. According to these mutations, various clinicalvariants have been described .PC type I (JadassohnLewandowsky, PC-I)consists of palmoplantar hyperkeratosis, follicular hyperkeratosis, and oralleukokeratosis. Occasionally, bullous lesions, hoarse voicedue to laryngeal involvement, warty lesions onknee and elbow, and hyperhidrosis may occur In PC type II (Jackson-Lawler, PC-II) . the palmoplantarkeratodermaand oral changes areof less importance or may be absent. In addition, history of natal teeth and the development of epidermal cysts or steatocysts areremarkable [6]

PC type III (Schafer-Brunauer, PC-III) . includescombined features of types 1 and 2 with angular chielitis, corneal dyskeratosis, andcataracts.Type IV includes features of type 1 and type 3 with laryngeal lesions, hoarseness of voicewith mental retardation, hair

\section{References}

1. StroberBE. Pachyonychiacongenit: type II. Dermatol Online J 2003;9:1: 2Muller C. On the causes of congenit onychogryphosis. Mcn Med Wocher 1904;49:2180-2. ^

3.Jadassohn J, Lewandowsky F. Jacol IkonographiaDermatologica. $1^{\text {st }}$ ed. Berlin: Urban und Schwarzenberg. Pachyonychiacongenita. Keratosis disseminatacircumscripta (follicular abnormalitiesand alopecia. Rare variants include pachyonychiacongenitatarda, characterized by isolated nail changesthat usually begin in the second and thirddecades of life [7]. These different presentations are currently known to be due to mutations in variable genes encoding one of thepaired epidermis keratins, $\mathrm{K} 6 \mathrm{a} / \mathrm{K} 16$ in PC-Iand $\mathrm{K} 6 \mathrm{~b} / \mathrm{K} 17$ in PCII [8].Complications like respiratory distress due tolaryngeal leucokeratosis and acroosteolysis,malignant changes in palmoplantar lesionscan occur in pachyonychiacongenita[9].In milder forms of pachyonychiacongenita,local emollients and keratolytics have beenused with considerable improvement. Oralretinoids have been demonstrated to improvethehyperkeratotic skin lesions. Retinoidsgiven for long periods produce a reasonabledegree of flattening of the nails [10]. The onlyeffective treatment for nail lesions is surgerywith radical excision of the nail, nail bed andnail matrix and skin implantation at the siteof improved nail. Surgical treatment is also important in case of oral lesions with hoarsenessor respiratory problems. When the familialmutation is known, genetic counseling can bedone and if required, prenatal diagnosis canbe done at early stage of pregnancy by chorionic villi biopsy [11]

Tylomata. Leukokeratosis linguae; : p. 29-31. \&

4Agarwal P, Chhaperwal MK, Sin A, Verma A, Nijhawan M, Singh al. Pachyonychiacongenita: A rar genodermatosis. Indian Dermato] Online J 2013;4:225-7. ^

[PUBMED] Full Text .

5. Johnson BL Jr, Yan AC. Congenital diseases (Geno- 
dermatosis). In: Elder DE । Lever's Histopathology of the Skin, 10th ed Philadelphia, Lippincott

Williams \& Wilkins, 2009: P 138

6. Su WP, Chun SI, Hammond Gordon H. Pachy-

onychiacongenita: A clinical stuc 12 cases and

review of the litera

PediatrDermatol 1990; 7: 33-

38. PMID: 2140447

7. Bansal A, Sethuraman G, Sh: VK. Pachyonychia

congenita with only

involvement. J Dermatol

2006; 33: 437-438. PMID: 167008

8.

Munro

Pachyonychiacongenita: Muta and

clinical presentations. $\mathrm{Br}$.

Dermatol 2001; 144:

929-930. PMID: 11359376
9. Murugesh SB, Reddy

Ragunatha S, et al. Acro-osteolysis: a complication of Jadas: Lewandowsky

syndrome. Int J Dermatol 2007 202- 205. PMID:

17269978

10. Lim TW, Paik JH, Kim N case of pachyonychia

congenita with oral leukoplakis steatocystoma

multiplex. J Dermatol 1999;

677-681. PMID:

10554435

11. Smith FJ, McKusick VA, Ni

$K$ et al., Cloning of

multiple keratin 16 genes facil prenatal diagno-

sis of pachyonychiacongenita ty PrenatDiagn

1999; 19: 941- 946. PMID: 10521 